\title{
B-Myb and C-Myb Play Required Roles in Neuronal Apoptosis Evoked by Nerve Growth Factor Deprivation and DNA Damage
}

\author{
David X. Liu, Subhas C. Biswas, and Lloyd A. Greene \\ Department of Pathology, Center for Neurobiology and Behavior and Taub Center for Alzheimer's Disease Research, Columbia University College of \\ Physicians and Surgeons, New York, New York 10032
}

Activation of cell cycle elements plays a required role in neuronal apoptosis associated with both development and neurodegenerative disorders. We demonstrated previously that neuron survival requires gene repression mediated by the cell cycle transcription factor E2F (E2 promoter binding factor) and that apoptotic stimuli lead to de-repression of E2F-regulated genes and consequent death. However, the downstream mediators of such death have been unclear. The transcription factors $B$ - and $C$ - $m y b$ are E2F-regulated genes that are induced in neurons by apoptotic stimuli. Here, we examine the role of B- and C-myb induction in neuron death. Antisense and siRNA constructs that effectively block the upregulation of B- and C-myb provide substantial protection against death of cultured neuronal PC12 cells, sympathetic neurons, and cortical neurons elicited by either NGF withdrawal or DNA damage. There is also significant protection from death induced by direct E2F-dependent gene de-repression. Our findings thus establish required roles for B- and C-myb in neuronal apoptosis.

Key words: Myb; E2F; NGF; neuron; apoptosis; cell cycle

\section{Introduction}

Neuronal apoptosis plays an indispensable role in neurogenesis and is an underlying cause for neurodegeneration. Recent findings suggest that apoptosis of postmitotic neurons is often associated with activation of the cell cycle machinery (Smith et al., 2000; Copani et al., 2001; Liu and Greene, 2001b; Becker and Bonni, 2004; Greene et al., 2004).

Abundant evidence has been presented that supports the following model for involvement of cell cycle molecules in neuron death (Park et al., 1998a,b; Liu and Greene, 2001b; Greene et al., 2004). In viable neurons, the cell-cycle-associated transcription factor E2F ( $\mathrm{E} 2$ promoter binding factor) is silenced by association with $\mathrm{Rb}$ family members and forms complexes that repress death-promoting genes. Apoptotic stimuli such as neurotrophic factor deprivation and DNA-damaging agents lead to activation of Cdk-4 and/or -6 (cyclin-dependent kinases), which in turn phosphorylate members of the $\mathrm{Rb}$ family. The latter phosphorylation event causes dissociation of E2F-Rb family repressor complexes and consequent de-repression of genes that lead to neuron death.

Genes with known susceptibility to E2F-dependent repression include the Myb transcription factor family members B-myb and

\footnotetext{
Received May 11, 2004; revised Aug. 20, 2004; accepted Aug. 24, 2004.

This work was supported in part by grants from the National Institutes of Health-National Institute of Neurological Disorders and Stroke and the Parkinson's Disease Foundation.

Correspondence should be addressed to David X. Liu, P\&S 15-401, Department of Pathology, Center for Neurobiology and Behavior and Taub Center for Alzheimer's Disease Research, Columbia University College of Physicians and Surgeons, New York, NY 10032. E-mail: d1345@columbia.edu.

D0I:10.1523/JNEUROSCI.1821-04.2004

Copyright $\odot 2004$ Society for Neuroscience $\quad$ 0270-6474/04/248720-06\$15.00/0
}

C-myb (Lam and Watson, 1993; Zwicker et al., 1996). Although B- and C-myb play important roles in cell cycle progression (Oh and Reddy, 1999), they have also been associated with proapoptotic activity (Selvakumaran et al., 1994; Bies and Wolff, 1995; Sala et al., 1996; Tashiro et al., 2004). With respect to nerve cells, C-myb transcripts are induced in sympathetic neurons by NGF withdrawal or exposure to a DNA-damaging agent (Estus et al., 1994; Besirli et al., 2003). Furthermore, we found that B- and $\mathrm{C}$-myb proteins are upregulated in neurons exposed to apoptotic stimuli and that this occurs via E2F-dependent gene derepression (Liu and Greene, 2001a). We further observed that overexpression of B- or C-myb is sufficient to induce death of cultured cortical neurons, sympathetic neurons, and neuronal (i.e., NGF-treated) PC12 cells. In light of these findings, we have investigated here whether B- and C-myb play required roles in neuron death.

\section{Materials and Methods}

Materials. Platinum TaqDNA polymerase and LipofectAMINE 2000 were from Invitrogen (San Diego, CA). Anti-human NGF and anti-GFP (green fluorescent protein) antisera, camptothecin, and Hoechst 33342 were from Sigma (St. Louis, MO). Anti-B-myb and anti-C-myb antibodies were from Santa Cruz Biotechnology (Santa Cruz, CA). Human recombinant NGF was a kind gift from Genentech (San Francisco, CA).

Cell culture. PC12 cells were cultured as described previously (Greene and Tischler, 1976). Neuronal PC12 cells were generated by exposure to NGF (100 ng/ml) in RPMI 1640 medium with 1\% horse serum for $7 \mathrm{~d}$. Embryonic rat cortical neurons and neonatal rat superior cervical ganglion (SCG) sympathetic neurons were cultured as described previously 
(Park et al., 1998b). For NGF deprivation, cell cultures were washed with NGF-free medium twice, and anti-NGF antibody (1:100) was added. Control cells were washed with serum-free medium and maintained in medium supplied with NGF without serum. HEK293 cells were cultured in DMEM with $10 \%$ fetal bovine serum.

Plasmids. Constructs and their kind sources were as follows: antisense B-myb (A. Sala, Institute of Child Health, London, UK and B. Calabretta, Thomas Jefferson University, Philadelphia, PA), antisense C-myb (G. Siu, Columbia University, New York, NY), E2F1(1-368) (W. Sellers, Harvard University, Boston, MA), and E2F1(1-374) (K. Helins, European Institute of Oncology, Milan, Italy). $B-m y b$ and $C-m y b$ siRNAs were prepared in the $p U 6$ vector using the GeneSuppressor System according to the instructions of the manufacturer (Imgenex, Sorrento Valley, VA) based on the sequences 5' - AAGGTCATTGAGCTGGTCAAG (B-myb) and 5'-AACACTTCCAGCAACCATGAA (C-myb). To convert human $B$ - and $C$ - $m y b$ into targets for siRNA against rat $B$ - and $C$ - $m y b$, we used the QuickChange Site-Directed Mutagenesis kit following the instructions of the manufacturer (Stratagene, La Jolla, CA), using primers $5^{\prime}$ GAGGAAGACCAAAAGGTCATTGAGCTGGTCAAGAAGTATGGCAC and 5' GTGCCATACTTCTTGACCAGCTCAATGACCTTTTGGTCTT-
CCTC for B-myb and primers 5'-TATAGATTCTTTCTTAAACACTTCCAGCAACCATGAAAACTCAGACTTGG and 5' -CCAAGTCTGAGTTTTCATGGTTGCTGGAAGTGTTTAAGAAAG-AATCTATA for $C-m y b$. Sequences of all genes generated from PCR were verified to be correct.

Transfections. DNA was prepared with Plasmid Maxi kits (Qiagen, Hilden, Germany). PC12 cells, cortical neurons, and SCG neurons were cotransfected with $0.4 \mu \mathrm{g}$ of $p C M S-E G F P, 0.2 \mu \mathrm{g}$ of antisense or siRNA construct against B-myb or C-myb, and $0.4 \mu \mathrm{g}$ of filler plasmid (pcDNA) or plasmid expressing E2F1(1-374) or E2F1(1-368) (when these constructs were used) per well in 24-well dishes using $1 \mu$ l of LipofectAMINE 2000. For Western blotting analysis, cycling PC12 cells were cotransfected with $1 \mu \mathrm{g}$ of $p C M S-E G F P$ and $1 \mu \mathrm{g}$ of control vector or antisense or siRNA construct against B-myb or C-myb per well in six-well dishes using $2 \mu \mathrm{l}$ of LipofectAMINE 2000. Transfection medium was maintained for $6 \mathrm{hr}$ and was then replaced with fresh complete medium. Camptothecin treatment $(10 \mu \mathrm{M})$, NGF withdrawal, and survival assays were initiated $24 \mathrm{hr}$ after transfection (day 0 ). To verify that the transfected cells in cortical cultures were indeed neurons, we stained transfected cultures in parallel experiments with monoclonal antibodies against neuron-specific tubulin (TuJ1), nestin, and GFAP. Among the transfected $\mathrm{GFP}^{+}$cells, 97\% (349 of 360) were
A.

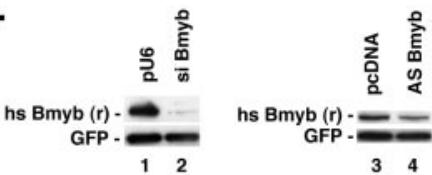

C.
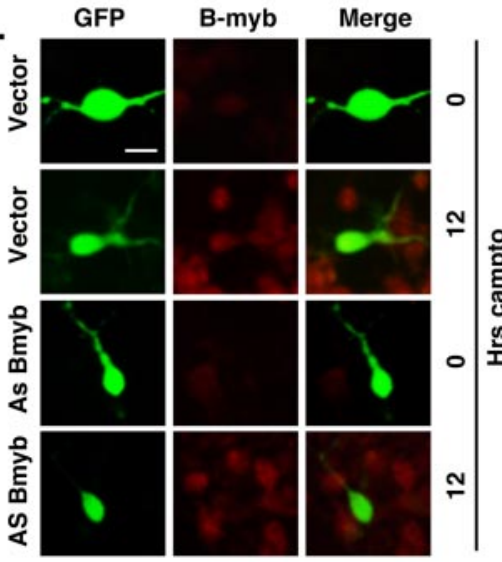

E.

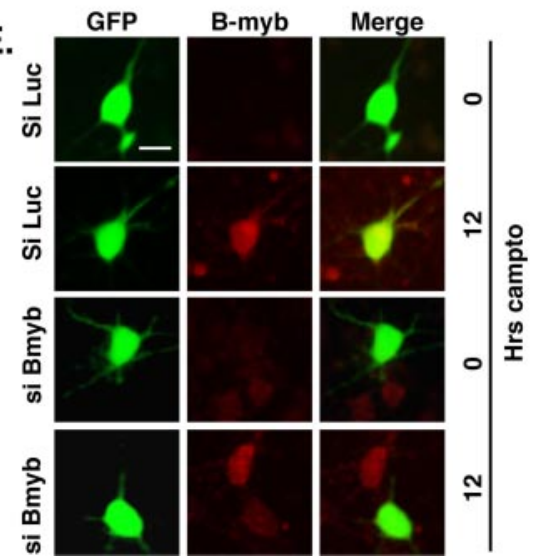

B.

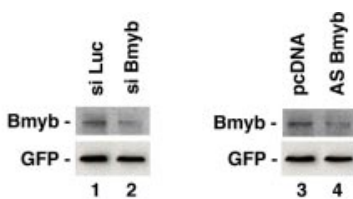

D.

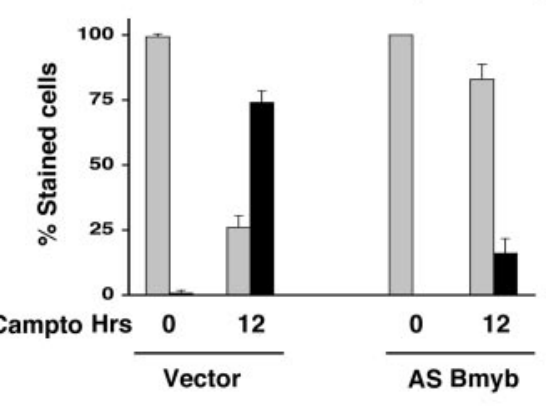

F.

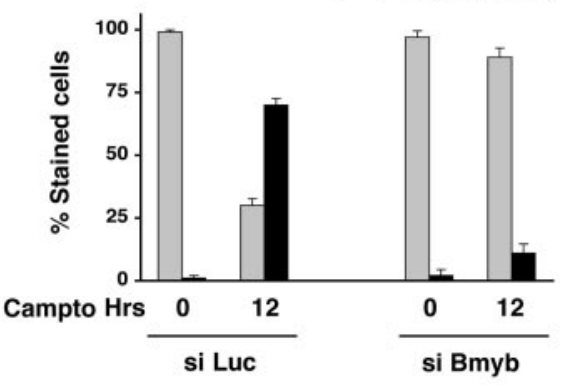

Figure 1. $\quad s i B$-myb and antisense $B$-myb constructs downregulate $B$-myb expression. $A$, Coexpression with either siB-myb or antisense $B$-myb downregulates expression in HEK293 cells of human/rat B-myb. pU6 and pCDNA are control, empty plasmids. $B$, Expression of either siB-myb or antisense B-myb downregulates endogenous B-myb expression in cycling PC12 cells. siluc and pcDNA are control plasmids. Transfection efficiency was $\sim 60-80 \%$. C, D, Antisense $B$-myb represses the upregulation of B-myb in cortical neurons treated with camptothecin (campto). Percentage of stained cells in this and subsequent figures pertains to the population of transfected neurons (as indicated by staining for GFP). E, F, siB-myb represses the upregulation of B-myb in cortical neurons treated with camptothecin. Scale bars, $10 \mu \mathrm{m}$.
$\mathrm{TuJ1}^{+}$, whereas $<1 \%$ were either nestin ${ }^{+}$or $\mathrm{GFAP}^{+}$. HEK293 cells were transfected as de-

Immunostaining. Immunostaining was performed as described by Angelastro et al. (2003). Briefly, cortical cultures were fixed with $4 \%$ paraformaldehyde-2\% sucrose in PBS for 15 min. After three washes in PBS, the cells were blocked in $10 \%$ non-immune goat serum and $0.3 \%$ Triton X-100 for $1 \mathrm{hr}$. The cultures were immunolabeled separately with the following combinations: (1) mouse anti-GFP (1:500; Sigma) and rabbit anti-B-myb (1:200; Santa Cruz Biotechnology), (2) mouse anti-GFP (1: $500)$ and rabbit anti-C-myb (1:200; Santa Cruz Biotechnology), (3) rabbit anti-GFP (1:1000; Clontech, Cambridge, UK) and mouse antiTuj1 (1:2000; Covance, Princeton, NJ), (4) rabbit anti-GFP (1:1000) and mouse anti-nestin (1:200; rat-401 from the Developmental Studies Hybridoma Bank antibody collection, University of Iowa, Iowa City, IA), or (5) rabbit anti-GFP (1:1000) and mouse antiGFAP (1:500; Chemicon, Temecula, CA) in $10 \%$ non-immune goat serum and $0.3 \%$ Triton X-100 overnight, followed by secondary labeling with goat FITC-conjugated antimouse and rhodamine-conjugated antirabbit antibodies (Alexa; Molecular Probes, Eugene, OR) at 1:5000 for combinations 1 and 2 , and with goat rhodamine-conjugated anti-mouse and FITC-conjugated anti-rabbit antibodies for combinations 3-5. Quantitative assessment of staining was performed in a blinded manner.

Western blotting. Western blotting was performed as described previously (Liu and Greene, 2001a).

Survival assays and counting of apoptotic nuclei. Survival assays and counting of apoptotic nuclei were as described previously (Liu and Greene, 2001a). Quantitative assessment of survival and death was performed in a blinded manner. All experiments were performed at least in triplicate, and results are reported as means \pm SEM. Student's $t$ test was performed as unpaired, two-tailed sets of arrays and presented as probability $(p)$ values. scribed previously (Liu and Greene, 2001a). 


\section{Results}

Apoptotic stimuli elevate endogenous $\mathrm{B}-$ and $\mathrm{C}-\mathrm{myb}$ in neurons, and this is blocked by B- and C-myb siRNA and antisense constructs

We reported previously that $B$ - and $C-m y b$ genes are repressed in viable neurons and de-repressed in dying neurons (Liu and Greene, 2001a). To investigate whether $\mathrm{B}$-myb plays a required role in neuronal apoptosis, we first prepared and examined the capacities of $s i B-m y b$ and antisense $B-m y b$ constructs to downregulate the expression of rat B-myb. As shown in Figure $1 A$, coexpression of $s i B-m y b$ (lane 2) effectively downregulated the expression in HEK293 cells of human B-myb that was mutated so that the siRNA target region matches the rat B-myb sequence (compare lane 2 with control lane 1 ). The capacity of the antisense $B-m y b$ to depress the level of coexpressed human/rat mutant $B-m y b$ was similarly confirmed in HEK293 cells (Fig. 1A, compare lane 4 with control lane 3 ). To examine the abilities of the two constructs to downregulate endogenous B-myb, we cotransfected them with $p C M S-E G F P$ (a control for transfection efficiency) into cycling PC12 cells and analyzed B-myb expression by Western blotting. As a control, empty vector was used in the case of antisense $B-m y b$ and a scrambled siRNA (siLuc) in the case of $s i B-m y b$. Approximately $60-80 \%$ of the cells were transfected under these conditions. As shown in Figure $1 B$, expression of either $s i B-m y b$ or antisense $B-m y b$ significantly reduced endogenous B-myb expression.

Next, we cotransfected $s i B-m y b$, antisense $B-m y b$, or a control vector together with $p C M S$-EGFP into cultured cortical neurons with or without $12 \mathrm{hr}$ exposure to the proapoptotic DNA-damaging agent camptothecin. The transfected cells were then immunostained to reveal B-myb expression. As illustrated in Figure $1 C-F$, for control cultures (vector alone or $s i L u c$ ), little or no staining was detectable without camptothecin exposure, whereas there was a high level of staining in $\sim 75 \%$ of the neurons after the treatment. This is consistent with our previous report that B-myb is upregulated in neurons in response to apoptotic stimuli (Liu and Greene, 2001a). We additionally found that antisense $B-m y b$ and $s i B$-myb each very significantly suppressed the induction of endogenous B-myb by camptothecin exposure (Fig. $1 C-F$ ). Similar observations were made for another apoptotic paradigm, NGF-deprivation in neuronal PC12 cell cultures (data not shown).

We used the same strategies to block induction of C-myb in neurons. As shown in Figure 2, siC-myb and antisense $C$ - $m y b$ both suppress expression of cotransfected rat/human mutant C-myb in HEK293 cells (Fig. 2A) and of endogenous C-myb in cycling PC12 cells (Fig. 2 B). Consistent with our previous report (Liu and Greene, 2001a), immunostaining revealed a substantial
B.

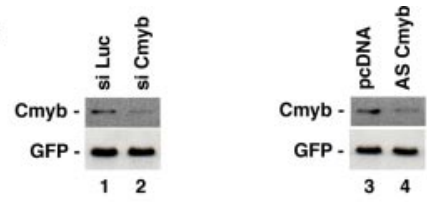

D.
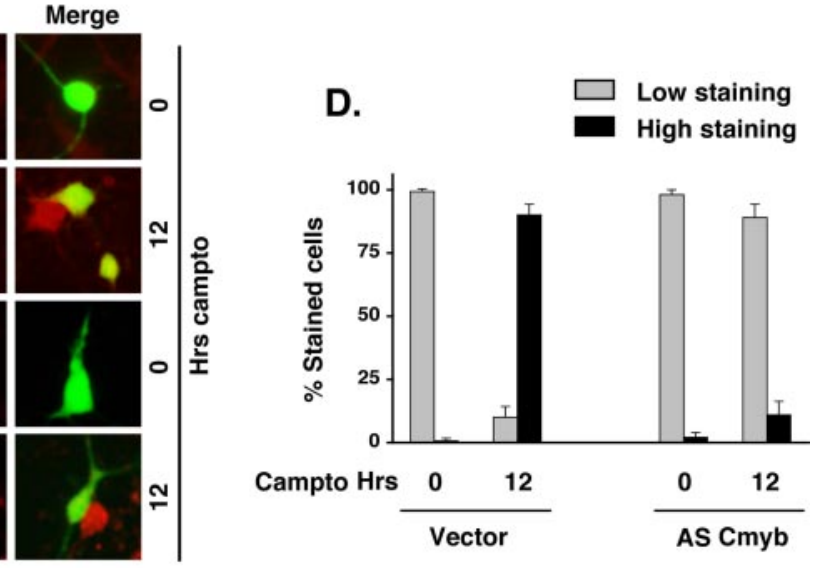

F.
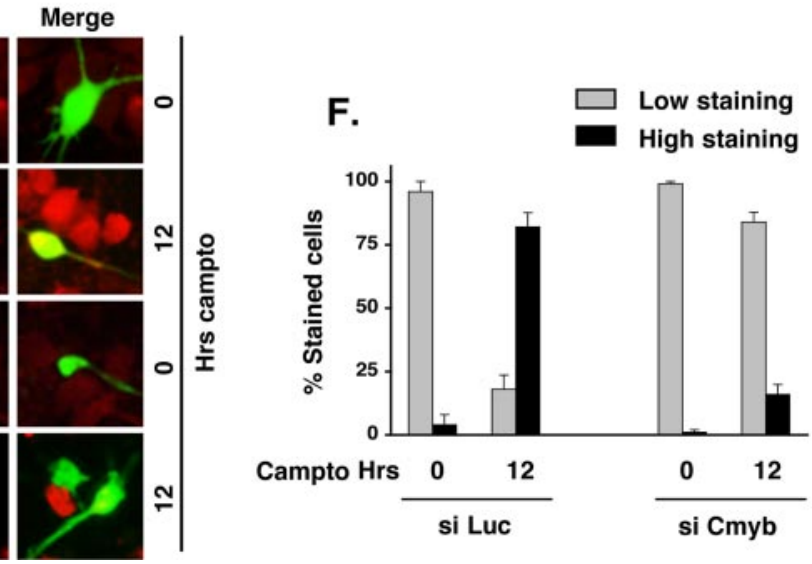

Figure 2. $\quad$ siC-myb and antisense C-myb constructs downregulate C-myb expression. $A$, Coexpression with either siC-myb or antisense C-myb downregulates expression in HEK293 cells of human/rat C-myb. B, Expression of either siC-myb or antisense $C-m y b$ represses the upregulation of $C$-myb in cortical neurons treated with camptothecin (campto). E, F, siC-myb represses the upregulation of C-myb in cortical neurons treated with camptothecin. Scale bars, $10 \mu \mathrm{m}$.

increase in endogenous C-myb expression in cultured cortical neurons after $12 \mathrm{hr}$ exposure to camptothecin (Fig. 2C-F). This increase was substantially suppressed by both antisense $C-m y b$ (Fig. 2C,D) and siC-myb (Fig. $2 E, F$ ). Here again, there were similar results in NGF-deprived PC12 cell cultures (data not shown).

\section{Downregulation of B-myb and C-myb promotes neuronal} survival in multiple apoptotic paradigms

We next examined the effects of B- and C-myb downregulation on neuronal cell survival in a variety of apoptotic paradigms. Figure $3 A$ shows that antisense $B-m y b$ and antisense $C-m y b$ each provided substantial protection against death evoked by NGF deprivation in PC12 cell cultures. Cotransfection of both antisense constructs increased survival almost to the level observed in the presence of NGF. $s i B-m y b$ and $s i C-m y b$ as well as the combination of the two also protected PC12 cells against NGF withdrawal (data not shown) and against camptothecin exposure (Fig. 3B). These constructs additionally suppressed death of cultured cortical neurons induced by camptothecin (Fig. 3C); similar results were obtained with antisense $B-m y b$ and antisense 

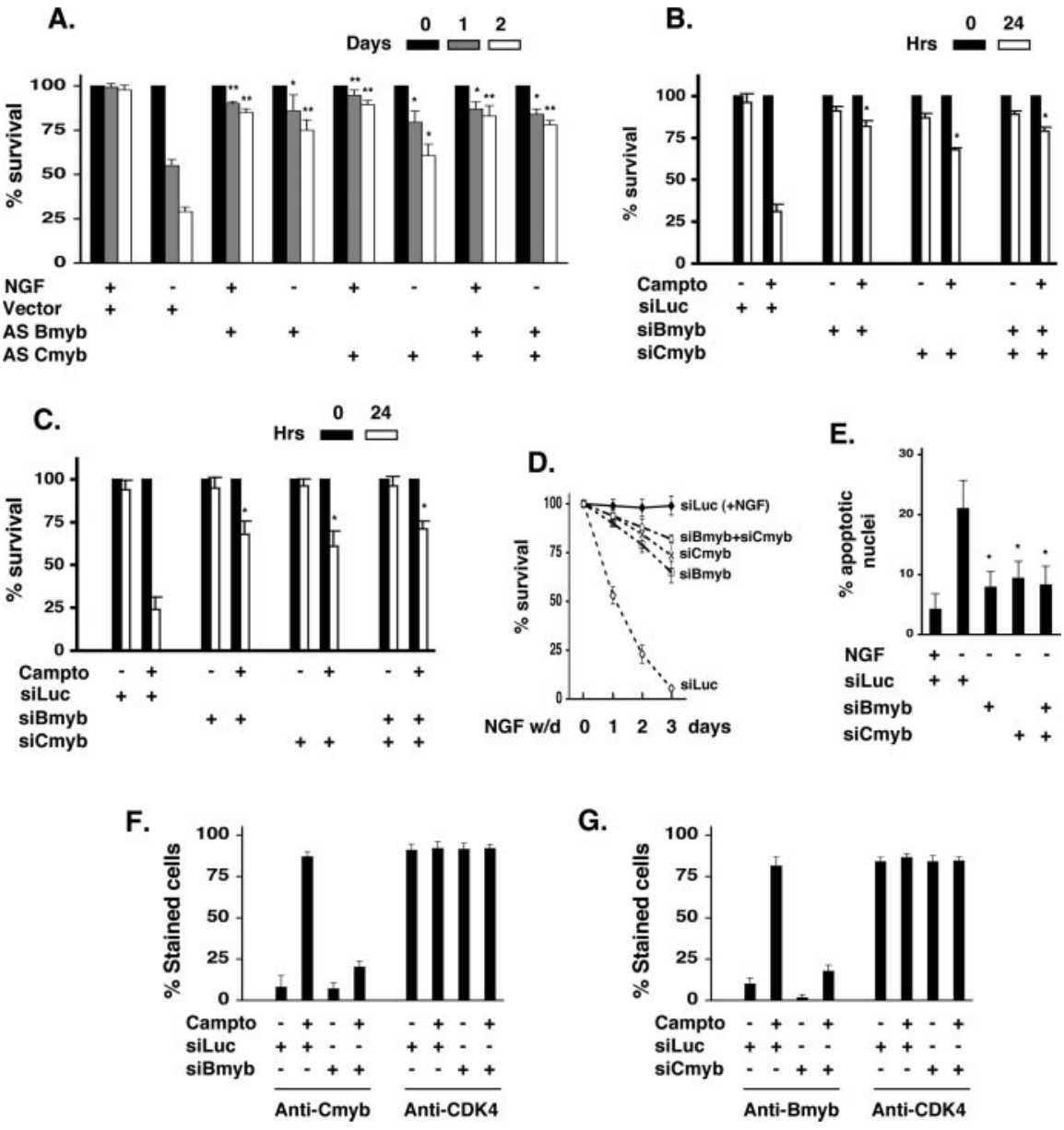

Figure 3. Downregulation of B-myb and C-myb protects neuronal cells from apoptotic stimuli. $A$, Expression of $B$ - and $C-m y b$ antisense constructs protects neuronal PC12 cells from death induced by NGF withdrawal. Significance comparisons with vector alone/NGF withdrawal on the same day, ${ }^{*} p<0.03$; ${ }^{* *} p<0.005$. B, Expression of siB-myb and siC-myb constructs protects neuronal PC12 cells from death induced by camptothecin (Campto) treatment. Significance comparisons with siluc control/ campthothecin, ${ }^{*} p<0.005$. C, Expression of siB-myb and siC-myb constructs protects cortical neurons from death induced by camptothecin treatment. Significance comparisons with siluc control/campthothecin, ${ }^{*} p<0.02$. D, E, Expression of siB-myb and siC-myb prevents death of sympathetic neurons induced by NGF withdrawal. $D$ indicates percentage of surviving neurons. NGF $\mathrm{w} / \mathrm{d}$, NGF withdrawal. Eshows percentage of transfected neurons with apoptotic nuclei on day 2 of NGF withdrawal. $E$, Significance comparisons with siluc/NGF withdrawal control, ${ }^{*} p<0.05$. F, siB-myb represses the upregulation of C-myb in cortical neurons treated with camptothecin. Cultures were transfected with siB-myb or siLuc and EGFP, treated with or without camptothecin for 12 $\mathrm{hr}$, and then stained for expression of C-myb and Cdk4 as indicated. G, siC-myb represses the upregulation of B-myb in cortical neurons treated with camptothecin. Cultures were transfected with siC-myb and EGFP, treated with or without camptothecin for $12 \mathrm{hr}$, and then stained for expression of B-myb and Cdk4 as indicated.

$C-m y b$ (data not shown). Downregulation of mybs with $s i B-m y b$ and $s i C-m y b$ (Fig. $3 D$ ) or antisense $B-m y b$ and antisense $C-m y b$ (data not shown) greatly enhanced survival of cultured sympathetic neurons for at least $3 \mathrm{~d}$ after NGF deprivation. This effect could also be seen by quantifying the numbers of apoptotic nuclei in transfected experimental and control cultures (Fig. $3 E$ and data not shown).

An intriguing aspect of our findings was that similar levels of protection were obtained with constructs designed to interfere with expression of either B-myb or C-myb. Moreover, in most instances, there was only an incremental increase in survival when reagents against both were used at the same time. B- and C-myb bind to similar DNA motifs (Rushton and Ness, 2001), and it has been reported that both B- and C-myb are subject to positive autoregulation (Nicolaides et al., 1991; Sala et al., 1999). Such observations raised the possibility that downregulation of

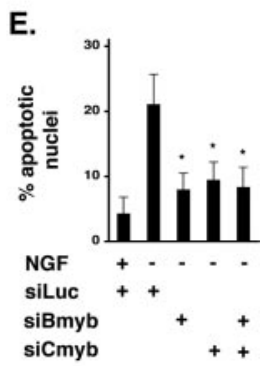

either B- or C-myb might cause crossdownregulation of the other. To test this, we repeated the experiments shown in Figure $1, E$ and $F$, in which cortical neurons were transfected with $s i B-m y b$ or $s i-$ Luc and treated with or without camptothecin for $12 \mathrm{hr}$. However, in this case, the cells were stained with antibodies against C-myb. As shown in Figure 3F, camptothecin greatly induced C-myb staining as anticipated. Significantly, siB-myb (but not siLuc) substantially repressed this induction. In contrast, expression of a control antigen (Cdk4) was not affected. Similar results were achieved when we performed the converse experiment of exposing the neurons to $s i C-m y b$ and staining for B-myb (Fig. 3G). These findings indicate that interfering with expression of either B- or C-myb results in downregulation of the other. Such observations appear to explain why siRNA or antisense constructs against either protein are equally effective.

\section{Downregulation of B-myb and C-myb mitigates the apoptotic effect caused by E2F de-repression}

We showed previously that E2F1(1-368), a truncation mutant of E2F1 lacking an activation domain, causes depression of myb expression in neurons and efficiently induces their death (Liu and Greene, 2001a). E2F1(1-374) (Phillips et al., 1997) is a similar E2F1 truncation mutant without an activation domain that also promotes de-repression of mybs (data not shown) and that induces death of cortical neurons and neuronal PC12 cells (Fig. 4). As shown in Figure $4 A$, expression of E2F1(1-374) brought about rapid death of neuronal PC12 cells so that only $\sim 20 \%$ survived $2 \mathrm{~d}$ after transfection and 3\% $3 \mathrm{~d}$ after transfection. Cotransfection with $s i B-m y b$ and $s i C-m y b$ increased survival to $\sim 60-70 \%$ on day 1 and $25 \%$ on day 2 . This increase in survival was mirrored by a substantial drop in the proportion of apoptotic nuclei present in the cultures (Fig. $4 B$ ). To extend this analysis to cortical neurons, E2F1(1-374) was cotransfected with either siLuc, siB-myb, or siC-myb. Again, E2F1(1-374) induced massive death, and this was significantly abrogated by myb downregulation (Fig. 4C). These findings support the idea that Band $\mathrm{C}-\mathrm{myb}$ are downstream mediators of death induced by E2F de-repression.

\section{Discussion}

Past and the present findings demonstrate that apoptotic stimuli such as NGF deprivation and DNA-damaging agents lead to elevated expression of B- and C-myb in both peripheral and CNS neurons (Estus et al., 1994; Liu and Greene, 2001a; Besirli et al., 2003). The major aim of the present study was therefore to assess whether B- and C-myb play required roles in these paradigms of neuron death. To this end, we prepared both antisense and 
A.
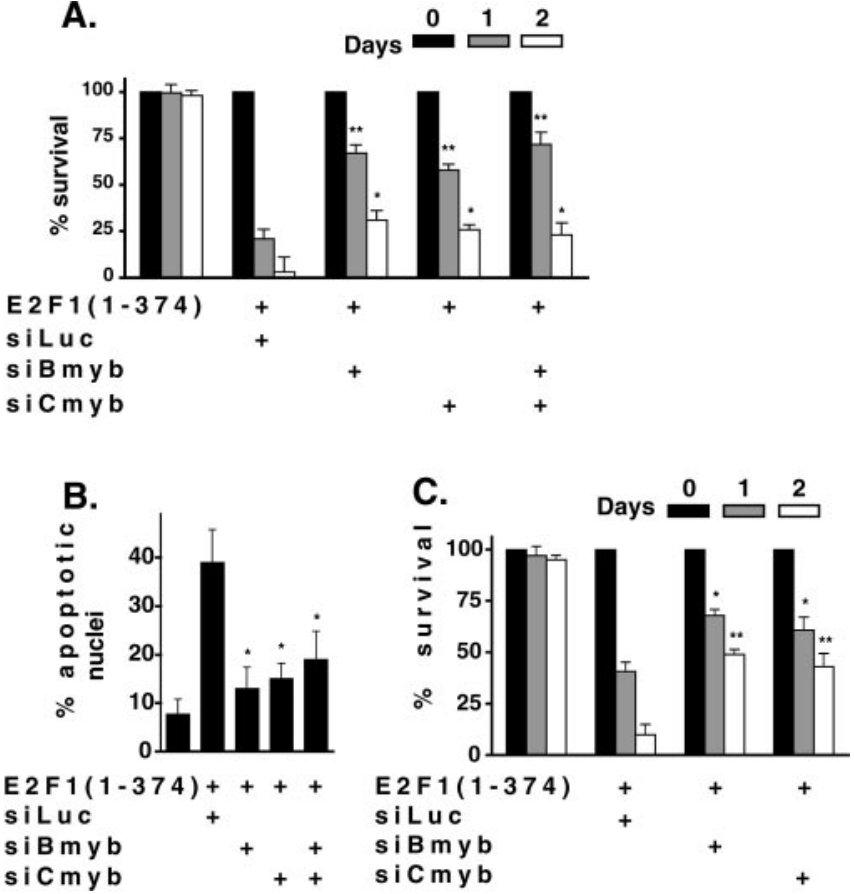

Figure 4. Downregulation of B-myb and C-myb protects neuronal cells from death caused by E2F de-repression. $A, B$, Coexpression of siB-myb or si $C$-myb increases survival $(A)$ and decreases apoptotic nuclei $(B)$ in neuronal $P C 12$ cells expressing E2F1(1-374). $A$, Significance comparisons with $\mathrm{E2F}(1-374)$ alone on the same day, ${ }^{*} p<0.02$; ${ }^{* *} p<0.005$. B, Significance comparisons with E2F1(1-374) alone, ${ }^{*} p<0.02 ;{ }^{* *} p<0.005$. C, Coexpression of $s i B-m y b$ or siC-myb promotes survival of cortical neurons expressing E2F1(1-374). Significance comparisons with siluc coexpressed with E2F1(1-374) as indicated on the same day, ${ }^{*} p<$ $0.01 ;{ }^{* *} p<0.002$.

siRNA constructs targeted to B- and C-myb and demonstrated that each approach effectively blocks induction of these proteins by apoptotic stimuli. We further observed that our constructs provided substantial protection in our apoptotic models, thus indicating that both $\mathrm{B}$ - and $\mathrm{C}-\mathrm{myb}$ are required for mediation of neuron death.

Although overexpression of B- or C-myb may induce death, it has also been reported that dominant-negative $(\mathrm{d} / \mathrm{n})$ forms of myb promote apoptosis of non-neuronal cells (Taylor et al., 1996; Lauder et al., 2001; Yi et al., 2002). We also found that $d / n$ myb induces neuron death (Liu and Greene, 2001a). Such findings raise the possibility that neuron survival may require an optimal level of myb expression; on one hand, elevated expression may promote apoptotic death, but on the other hand, a basal level of expression may be required for viability. Consistent with this, we detected both B- and C-myb in viable neurons (Liu and Greene, 2001a), and C-myb is widely and constitutively expressed in brain (Shin et al., 2001). Moreover, we observed neuron death with application of myb antisense and siRNA constructs at concentrations as little as 2.5-fold higher than those that effectively protected neurons from apoptotic stimuli (our unpublished results). In this regard, it may be relevant that Bcl-2 has been found to be a target of both C-myb (Wolff et al., 2001) and B-myb (Grassilli et al., 1999).

$\mathrm{B}$ - and C-myb are subject to E2F-dependent gene repression and are induced during neuron death by a mechanism that appears to require Cdk4/6-promoted dissociation of Rb family-E2F complexes and consequent gene de-repression (Liu and Greene, 2001a,b; Greene et al., 2004). As one test of this mechanism, we found that neuron death induced by E2F1(1-374), a truncation mutant lacking transactivational activity, was significantly reduced by interfering with B- and C-myb expression. The lack of full protection under these circumstances may reflect the participation of additional E2F-regulated genes in neuron cell death or of nonphysiologic apoptotic actions of the overexpressed E2F proteins.

In summary, our findings support a major required role for Band C-myb as downstream mediators of neuronal cell death evoked by NGF deprivation and DNA damage. Mybs have been proposed as novel targets for cancer therapy; our results suggest that such targeting may also be considered for therapeutic blockade of neuron cell death.

\section{References}

Angelastro JM, Ignatova TN, Kukekov VG, Steindler DA, Stengren GB, Mendelsohn C, Greene LA (2003) Regulated expression of ATF5 is required for the progression of neural progenitor cells to neurons. J Neurosci 23:4590-4600.

Becker EB, Bonni A (2004) Cell cycle regulation of neuronal apoptosis in development and disease. Prog Neurobiol 72:1-25.

Besirli CG, Deckwerth TL, Crowder RJ, Freeman RS, Johnson Jr EM (2003) Cytosine arabinoside rapidly activates Bax-dependent apoptosis and a delayed Bax-independent death pathway in sympathetic neurons. Cell Death Differ 10:1045-1058.

Bies J, Wolff L (1995) Acceleration of apoptosis in transforming growth factor beta 1-treated M1 cells ectopically expressing B-myb. Cancer Res 55:501-504.

Copani A, Condorelli F, Canonico PL, Nicoletti F, Sortino MA (2001) Cell cycle progression towards Alzheimer's disease. Funct Neurol 16:11-15.

Estus S, Zaks WJ, Freeman RS, Gruda M, Bravo R, Johnson Jr EM (1994) Altered gene expression in neurons during programmed cell death: identification of c-jun as necessary for neuronal apoptosis. J Cell Biol 127:1717-1727

Grassilli E, Salomoni P, Perrotti D, Franceschi C, Calabretta B (1999) Resistance to apoptosis in CTLL-2 cells overexpressing B-Myb is associated with B-Myb-dependent bcl-2 induction. Cancer Res 59:2451-2456.

Greene LA, Tischler AS (1976) Establishment of a noradrenergic clonal line of rat adrenal pheochromocytoma cells which respond to nerve growth factor. Proc Natl Acad Sci USA 73:2424-2428.

Greene LA, Biswas SC, Liu DX (2004) Cell cycle molecules and vertebrate neuron death: E2F at the hub. Cell Death Differ 11:49-60.

Lam EW, Watson RJ (1993) An E2F-binding site mediates cell-cycle regulated repression of mouse B-myb transcription. EMBO J 12:2705-2713.

Lauder A, Castellanos A, Weston K (2001) c-Myb transcription is activated by protein kinase $\mathrm{B}(\mathrm{PKB})$ following interleukin 2 stimulation of Tcells and is required for PKB-mediated protection from apoptosis. Mol Cell Biol 21:5797-5805.

Liu DX, Greene LA (2001a) Regulation of neuronal survival and death by E2F-dependent gene repression and derepression. Neuron 32:425-438.

Liu DX, Greene LA (2001b) Neuronal apoptosis at the G1/S cell cycle checkpoint. Cell Tissue Res 305:217-228.

Nicolaides NC, Gualdi R, Casadevall C, Manzella L, Calabretta B (1991) Positive autoregulation of c-myb expression via Myb binding sites in the 5 ' flanking region of the human c-myb gene. Mol Cell Biol 11:6166-6176.

Oh IH, Reddy EP (1999) The myb gene family in cell growth, differentiation and apoptosis. Oncogene 18:3017-3033.

Park DS, Morris EJ, Padmanabhan J, Shelanski ML, Geller HM, Greene LA (1998a) Cyclin-dependent kinases participate in death of neurons evoked by DNA-damaging agents. J Cell Biol 143:457-467.

Park DS, Morris EJ, Stefanis L, Troy CM, Shelanski ML, Geller HM, Greene LA (1998b) Multiple pathways of neuronal death induced by DNAdamaging agents, NGF deprivation, and oxidative stress. J Neurosci $18: 830-840$

Phillips AC, Bates S, Ryan KM, Helin K, Vousden KH (1997) Induction of DNA synthesis and apoptosis are separable functions of E2F-1. Genes Dev 11:1853-1863.

Rushton JJ, Ness SA (2001) The conserved DNA binding domain mediates similar regulatory interactions for A-Myb, B-Myb, and c-Myb transcription factors. Blood Cells Mol Dis 27:459-463.

Sala A, Casella I, Grasso L, Bellon T, Reed JC, Miyashita T, Peschle C (1996) 
Apoptotic response to oncogenic stimuli: cooperative and antagonistic interactions between c-myb and the growth suppressor p53. Cancer Res 56:1991-1996.

Sala A, Saitta B, De Luca P, Cervellera MN, Casella I, Lewis RE, Watson R, Peschle C (1999) B-MYB transactivates its own promoter through SP1binding sites. Oncogene 18:1333-1339.

Selvakumaran M, Lin HK, Sjin RT, Reed JC, Liebermann DA, Hoffman B (1994) The novel primary response gene $\mathrm{MyD} 118$ and the proto-oncogenes myb, myc, and bcl-2 modulate transforming growth factor beta 1-induced apoptosis of myeloid leukemia cells. Mol Cell Biol 14:2352-2360.

Shin DH, Lee HW, Jeon GS, Lee HY, Lee KH, Cho SS (2001) Constitutive expression of c-myb mRNA in the adult rat brain. Brain Res 892:203-207.

Smith DS, Leone G, DeGregori J, Ahmed MN, Qumsiyeh MB, Nevins JR (2000) Induction of DNA replication in adult rat neurons by deregula- tion of the retinoblastoma/E2F G1 cell cycle pathway. Cell Growth Differ 11:625-633.

Tashiro S, Sumi T, Uozumi N, Shimizu T, Nakamura T (2004) B-Mybdependent regulation of c-Myc expression by cytosolic phospholipase A2. J Biol Chem 279:17715-17722.

Taylor D, Badiani P, Weston K (1996) A dominant interfering Myb mutant causes apoptosis in T cells. Genes Dev 10:2732-2744.

Wolff L, Schmidt M, Koller R, Haviernik P, Watson R, Bies J, Maciag K (2001) Three genes with different functions in transformation are regulated by c-Myb in myeloid cells. Blood Cells Mol Dis 27:483-488.

Yi HK, Nam SY, Kim JC, Kim JS, Lee DY, Hwang PH (2002) Induction of apoptosis in K562 cells by dominant negative c-myb. Exp Hematol 30:1139-1146.

Zwicker J, Liu N, Engeland K, Lucibello FC, Muller R (1996) Cell cycle regulation of E2F site occupation in vivo. Science 271:1595-1597. 